\title{
Synchrotron radiation CT methods for 3D quantitative assessment of mechanically relevant ultrastructural properties in murine bone
}

\author{
Philipp Schneider*a ${ }^{*}$ Romain Voide ${ }^{\mathrm{a}}$, Marco Stampanoni ${ }^{\mathrm{b}}$, Ralph Müller ${ }^{\mathrm{a}}$ \\ ${ }^{a}$ Institute for Biomechanics, ETH Zürich, Wolfgang-Pauli-Strasse 10, 8093 Zürich, Switzerland \\ ${ }^{\mathrm{b}}$ Swiss Light Source (SLS), Paul Scherrer Institut (PSI), 5232 Villigen, Switzerland
}

\begin{abstract}
Recent data have shown that predicting bone strength can be greatly improved by including microarchitectural parameters in the analysis. Moreover, bone ultrastructure has been implicated as an important contributor to bone strength. We therefore hypothesized that a better understanding of phenotypes linked to bone ultrastructure will provide new insight in the assessment of bone quality and its contribution to bone strength and fracture risk. Therefore, we first developed an experimental design to assess quantitatively ultrastructural murine bone tissue properties non-invasively in three dimensions by using synchrotron radiation-based (SR) computed tomography (CT) methods with resolutions on the order of one micrometer and below. New morphometric indices were introduced to quantify ultrastructural phenotypes of murine cortical bone assessed by our SR CT-based setup, namely the canal network and the osteocyte lacunar system. These ultrastructural phenotypes were then successfully studied in two genetically distinct mouse strains. Finally, we provided strong evidence for a significant influence of the canal network on murine bone mechanics. In the long run, we believe that the morphometric analysis of the ultrastructural phenotypes and the study of bone phenotypes at different hierarchy levels, in conjunction with bone mechanics, will provide new insights in the assessment of bone quality.
\end{abstract}

Keywords: Bone ultrastructure, canal network, osteocyte lacunae, cortical bone, mouse model, computed tomography, synchrotron radiation, negative imaging, morphometry, bone mechanics

\section{INTRODUCTION}

Bone mineral density (BMD), a calibrated measure of bone mass, has been identified in several epidemiological studies as being the single most important risk factor for osteoporotic fractures ${ }^{1,2}$. Hence, research efforts over the past three decades have focused on defining major determinants of bone mass and bone loss. Nevertheless, the agreement that BMD is the best predictor of fracture risk has become a controversial issue ${ }^{3}$. For example, it has been shown that, on an individual basis, density alone accounts for 10 to $90 \%$ of the variation in the strength of trabecular bone ${ }^{4}$. This also means that up to $90 \%$ of the variation in strength cannot be explained by bone density alone ${ }^{5}$. Recent data have shown that predicting bone strength can be greatly improved by including microarchitectural parameters in the analysis ${ }^{6,7}$. Moreover, the canal network has been shown to be an important contributor to local tissue porosity ${ }^{8-10}$, and is therefore important for the mechanical properties of bone. We consequently hypothesized that a better understanding of phenotypes linked to bone ultrastructure will provide new insight in the assessment of bone quality and its contribution to bone strength and fracture risk, and eventually that prediction of bone strength can be improved by including ultrastructural bone tissue properties. To test this hypothesis, the following specific aims were proposed:

1) Cannular and cellular imaging of bone (section 2): Development of an experimental setup to assess quantitatively ultrastructural murine bone tissue properties non-invasively in three dimensions (3D) by using computed tomography (CT) methods with resolutions on the order of one micrometer and below.

2) Structure function relationships in murine bone (section 3): Study of ultrastructural phenotypes to identify and evaluate genetic predispositions of these phenotypes and their relevance for bone mechanics.

For vascular and cellular imaging of bone, a synchrotron radiation (SR)-based local CT method was devised for the assessment of hard tissues in the submicrometer domain (section 2.1). In a next step, new morphometric indices were introduced to quantify ultrastructural phenotypes of murine cortical bone assessed by SR CT, namely the canal network

*pschneider@ethz.ch; phone + 4144632 4569; fax +41 44633 1124; http://www.biomechanics.ethz.ch

Medical Imaging 2008: Physiology, Function, and Structure from Medical Images

edited by Xiaoping P. Hu, Anne V. Clough, Proc. of SPIE Vol. 6916, 691619, (2008)

1605-7422/08/\$18 - doi: 10.1117/12.772668

Proc. of SPIE Vol. 6916 691619-1 
and the osteocyte lacunar system (section 2.2). The power of the mouse as animal model is manifold. First, there exists a high homology between the human and mouse genome. Moreover, the mouse genome has become highly defined among the various inbred strains, which greatly facilitates rapid location of new genes. Second, mice of a given inbred strain represent unlimited numbers of genetically identical twins whose genes can be analyzed experimentally and - equally important - each inbred strain is genetically different from every other inbred strain, allowing planned matings and studies of segregating genes affecting the development and maintenance of bone. Finally, the relative control that investigators have over environmental factors readily facilitates their separation from genetic factors. The last part of the present work is dedicated to the examination of structure function relationships in murine bone, where interactions between macroscopic, ultrastructural, and mechanical properties were identified and evaluated (section 3.1).

\section{CANNULAR AND CELLULAR IMAGING OF BONE}

\subsection{Synchrotron CT for hard tissue assessment in the submicrometer domain}

\section{Introduction}

Traditionally, bone architecture has been studied by optical microscopy, based on two-dimensional (2D) histomorphometry. Classical bone histomorphometry involves embedding and serial sectioning of the sample, which is laborious as well as destructive, and additionally, implies model assumptions for the computation of 3D morphometric indices, as it is inherently a $2 \mathrm{D}$ technique. In comparison ${ }^{11}$, CT overcomes these limitations, since it is a non-destructive and truly 3D technique. Moreover, the continuously increasing spatial resolution capabilities of CT systems provide a more detailed insight into bone tissue. Currently, SR facilities offer CT systems running at spatial resolutions down to around $1 \mu \mathrm{m}^{12}$ and novel techniques are under development ${ }^{13}$ for CT even below $100 \mathrm{~nm}$. However, SR CT systems are only rarely used close to their full resolution capacity for the examination of biological specimens ${ }^{14-16}$. This is primarily due to the fact that for a given charge coupled device (CCD) with a fixed pixel array, every increase in magnification of the optical system is accompanied by an equal reduction in the fraction of the recorded sample projection, i.e. by a decrease in the field of view (FOV). In other words, the maximal sample size that can be assessed by current SR CT setups in their standard mode is limited by the magnification of the optical system. Namely, the most popular category of CCD cameras presently in use for SR CT applications ${ }^{12}$ are equipped with an image area of up to $2048 \times 2048$ pixels and thus, restrict the sample diameter to less than $1 \mathrm{~mm}$ in cross section for observations at a theoretical pixel size below 0.5 $\mu \mathrm{m}$ or for a corresponding spatial resolution on the order of $1 \mu \mathrm{m}$, respectively. Since intact (biological) specimens are typically larger than $1 \mathrm{~mm}$, this constraint prevents non-destructive investigations in the micrometer and submicrometer regime. Here we present a strategy, where we developed a non-destructive local SR CT method operating in the submicrometer regime, which can be implemented for already existing experimental stations dedicated to SR CT.

\section{Methods}

A beagle dog was raised at Indiana University School of Medicine's (IUSM) AALAC-accredited facility (Indianapolis, IN, USA) and was sacrificed at the age of one year. The cranial portion of the third lumbar vertebra (L3) of the dog was dissected and stored for analysis. All animal procedures were approved by the IUSM Animal Care and Use Committee.

The SR CT measurement was performed in air at the X-ray Tomographic Microscopy (XTM) station ${ }^{17}$ of the Materials Science (MS) beamline ${ }^{18}$ at the Swiss Light Source (SLS). A total of 1001 projections were acquired over a range of 180 degrees at a photon energy of $17.5 \mathrm{keV}$ and at a theoretical pixel size of $350 \mathrm{~nm}$. To increase the signal-to-noise ratio (SNR), the data was additionally binned twice, resulting in a theoretical pixel size of $700 \mathrm{~nm}$. The projections were reconstructed using filtered backprojection (FBP).

In our local CT setup, the specimen was bigger than the FOV perpendicular to the rotation axis and therefore, only a portion of the whole sample was assessed. This allowed for high-resolution CT without destruction of the specimen. However, the major problem with local CT measurements is the loss of information required by theory for a proper reconstruction of the specimen's density function, which is a direct consequence of the partial recording of the sample projections. In contrast, the sample projections are captured entirely in the case of a standard (i.e. global) CT setup, and correct reconstruction of the specimen is feasible ${ }^{19}$. On this account, several mathematical local tomographic methods have been derived to localize the reconstruction ${ }^{20}$. However, these local tomographic methods never reconstruct the original density function of the specimen, but only related functions which behave similarly. Especially for hard tissues, i.e. for highly absorbing X-ray materials such as bone, residual contrast errors (contrast anomalies) within the reconstructed images can hamper their interpretation ${ }^{21}$. Furthermore, mathematical local tomographic methods have not 
been established as a standard method up to now, such as the conventional FBP for global $\mathrm{CT}^{22}$. For these reasons, they have been used only infrequently for medical imaging. In this study, we introduce a pragmatic local tomography method correcting for cupping ${ }^{23}$ as the most prominent contrast anomaly in the reconstructions obtained from FBP. Cupping refers to the effect that for a local CT, fractions of the sample outside of the FOV are not reconstructed, but add image energy during FBP, which finally lead to a radial increase of the gray values towards the rim of the reconstruction circle. This effect is shown in Figure 1A, where we measured the trabecular compartment of the canine lumbar vertebra with our local SR CT setup at a theoretical pixel size of $350 \mathrm{~nm}$, including subsequent two-fold binning.
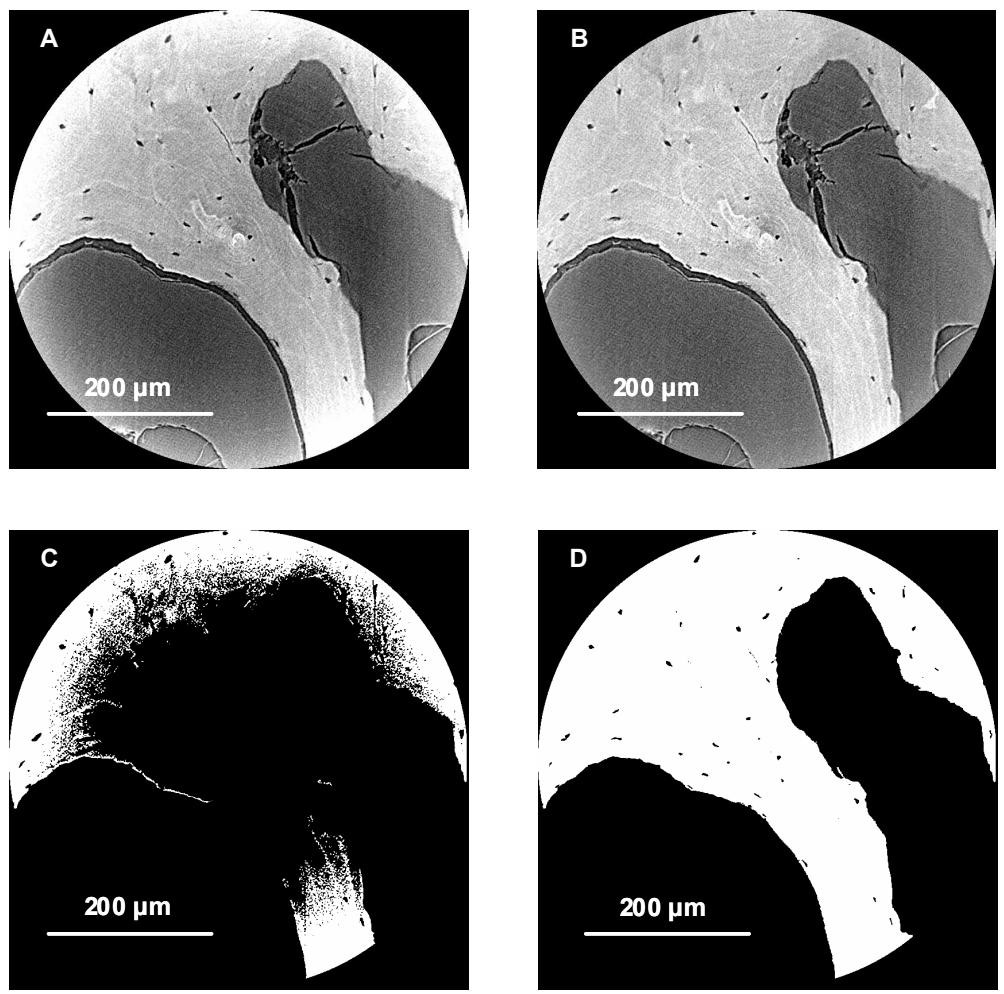

Figure 1. Radial gray value profile correction for local synchrotron radiation (SR)-based CT. A: Reconstructed slice of the trabecular compartment of a canine lumbar vertebra. The effect of increased gray values for larger radial distances from the reconstruction center (midpoints) is called "cupping" and is due to the local tomographic measurement setup. $B$ : Reconstructed slice after radial polynomial gradient (RPG) correction. The radial gray value profile became flat compared to the original reconstruction shown in subfigure A. C: Original reconstructed slice after global thresholding. Apparently, segmentation by global thresholding was not successful due to cupping. $D$ : Corrected slice after global thresholding. Bone segmentation using global thresholding was now feasible due to the implemented RPG correction. $A-D$ : Data has been assessed by local SR CT at $350 \mathrm{~nm}$ theoretical pixel size with subsequent two-fold binning.

For $3 \mathrm{D}$ analysis and visualization, segmentation of the gray-scale image data is necessary. Nevertheless, typical segmentation schemes such as global thresholding fail when cupping is present (Figure 1C). As a consequence, morphometrical quantification of the binarized bone ultrastructure is no longer feasible. There is no theoretical basis for determining analytically the necessary cupping correction ${ }^{24}$ when using local tomography reconstruction theory ${ }^{20,23,24}$. For this reason, we heuristically quantified the cupping effect by calculating the gray value of the bone tissue depending on the distance from the reconstruction center (midpoints of Figures 1A-D) and by averaging the obtained values for 256 consecutive reconstructed slices.

\section{Results}

The resulting gray value profile (denoted as Original in Figure 2$)$ followed a second degree polynomial $\left(R^{2}=0.99\right)$. In a next step, we processed the reconstructed canine trabecular bone compartment applying the radial polynomial gradient (RPG) correction, as illustrated in Figure 1B. In comparison with the original reconstruction (Figure 1A), the radial gray value profile became flat, which is demonstrated in Figure 2. Finally, global thresholding yielded accurate segmentation as demonstrated in Figure 1D and allowed for potential quantitative morphometric analysis of the bone ultrastructure. 


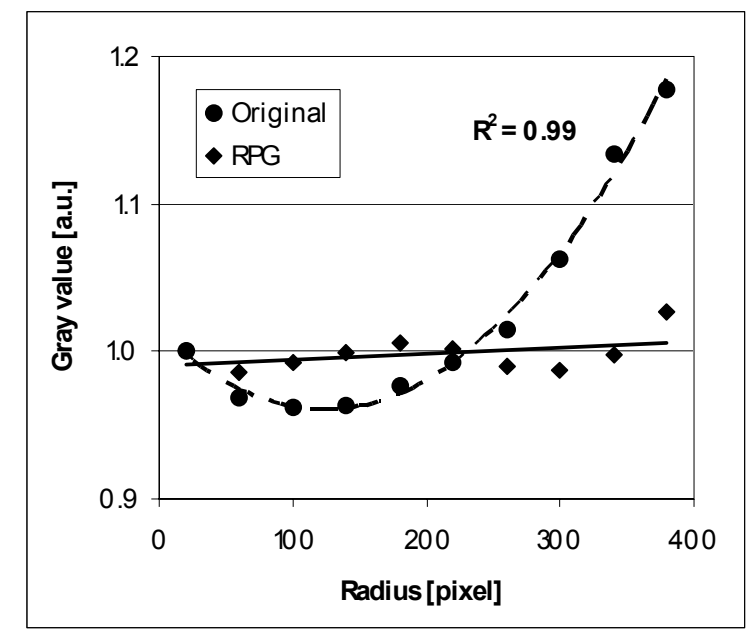

Figure 2. Quantification of the radial gray value profile within reconstructed bone tissue assessed by local synchrotron radiation (SR)-based CT. The figure shows the radial gray value profile for the original reconstructed data set of the trabecular compartment of a canine lumbar vertebra shown in Figure 1A and for the same data set after radial polynomial gradient (RPG) correction depicted in Figure 1B. The radial gray value gradient profile of the original tomographic data set (Original) described by a second degree polynomial became flat after RPG correction $(R P G)$.

\section{Conclusions}

As a novelty, the developed CT method overcame present experimental limitations with respect to object size, currently precluding conventional CT methods from hard tissue assessment in the submicrometer domain. In particular, a correction algorithm for SR-based local CT has been devised which tackles the existing object size limitation encountered in conventional CT setups for submicrometer imaging of hard tissues. Our method offers a strategy for SRbased CT assessment and quantitative analysis of intact specimens larger than the reconstruction circle in a truly 3D fashion and at unprecedented resolutions in the submicrometer regime.

\subsection{Ultrastructural properties in murine cortical bone}

\section{Introduction}

Recent results show that the canal network is a major contributor to local tissue porosity ${ }^{8-10}$, and therefore, can directly be linked to measures of bone tissue quality and thus, to the mechanical properties of bone. Additionally, osteocyte lacunae are believed to act as stress concentrators within tissue of compact bone ${ }^{25}$. Therefore, the purpose of this study was to establish a strategy to subdivide murine intracortical porosity into 3D ultrastructural phenotypes, namely the canal network and the osteocyte lacunar system, and to provide methods to volumetrically quantify these phenotypes for subsequent morphometric analysis.

Within this study, we followed a hierarchical description of the cortical bone matrix ${ }^{26}$. For these purposes, we distinguished three levels on different length scales. On the organ level, cortical bone was considered to be compact and was characterized by its radial extension and volume distribution. On the tissue level and cellular level, we described the ultrastructure of the intracortical porosity. Whereas the tissue level was described by the cannular network, the osteocyte lacunar system constituted the cellular level. Here, we first (Methods) describe the imaging setup and the subsequent image analysis facilitating ultrastructural bone morphometry in a generic fashion. In a second part (Application) the animal model and the specific aims of the genetic study are presented in detail.

\section{Methods}

To study the organ and tissue level of the cortical bone, we first scanned the femoral mid-diaphysis of 4-month-old mouse using a conventional (i.e., global) SR CT setup at a nominal resolution of $3.5 \mu \mathrm{m}$. This cortical compartment started at $56 \%$ of the whole femur length (calculated from the greater trochanter) according to Kohler et al. ${ }^{27}$ and contained 388 slices resulting in a stack height of $1.56 \mathrm{~mm}$. To study the cellular level, we applied additionally local SR 
$\mathrm{CT}$ at a submicrometer resolution (700-nm nominal resolution) in a second step. Using this local imaging setup, the femur was scanned at the anterior, posterior, lateral, and medial site of the mid-diaphysis. The FOV within the cortical bone assessed by the local SR CT measurements was a subset of the FOV of the global SR CT setup, so that the same cortical region was imaged and corresponding ultrastructural morphometric parameters could be assessed. The SR CT measurements were performed in air at the X-ray Tomographic Microscopy (XTM) station ${ }^{17}$ of the Materials Science (MS) beamline at the Swiss Light Source (SLS) ${ }^{18}$. For both setups, a total of 1001 projections was acquired over a range of $180^{\circ}$ at a photon energy of $17.5 \mathrm{keV}$. The data were reconstructed using filtered backprojection.

A new sinogram-based algorithm was devised to eliminate ring artifacts, which arose from defects on the scintillator of the optical system at the XTM station and which were clearly visible in the reconstructed slices. To partially suppress noise within the reconstructed tomograms a constrained Gaussian filter was applied. Subsequent iterative global thresholding ${ }^{28}$ provided binarized data sets separating bone matrix from soft tissue and air. For quantitative analysis of the bone tissue on the organ level, component labeling and morphologic operations were applied to the binarized data to obtain a solid representation of the mid-diaphysis. The technique of negative imaging was applied in this study to assess the porosity within cortical bone on the tissue and on the cellular level. In this context, negative imaging denotes the technique to first measure the matrix of a porous structure using CT, and subsequently, to extract the enclosed porosity as a negative imprint of the surrounding matrix. In this study, the extraction of the cortical void spaces comprised different image processing procedures (IPL; Scanco Medical AG, Brüttisellen, Switzerland), including additional morphological operators. They were optimized to extract the canal network and the osteocyte lacunar system within cortical bone as two separate phases.

For morphometry on the organ level, standard 3D algorithms ${ }^{29}$ were used to compute total tissue volume (TV) enclosing both the medullary cavity and the cortical bone tissue, cortical bone volume (Ct.BV), cortical bone volume density (Ct.BV/TV), cortical thickness (Ct.Th), and polar area moment (J). On the tissue level, the void spaces within cortical bone obtained by negative imaging formed cannular structures. We believe that these cannular elements represent the living space of the vasculature and/or bone remodeling units (including osteoclast lacunae) within cortical bone. We name this phenotype canal network and we precede each corresponding parameter the designation canal or cannular. For quantification of the canal network, we introduced cannular indices, corresponding to standard nomenclature of bone morphometry ${ }^{30}$ for trabecular bone and similar to the naming of cannular parameters introduced by Cooper et al. ${ }^{10}$. Hence, we defined number of canals (N.Ca), canal number density (N.Ca/Ct.TV), canal volume (Ca.V), canal volume density (Ca.V/Ct.TV), and canal spacing (Ca.Sp), where Ct.TV designates the cortical total volume. These parameters describe the overall canal network and they were quantified by standard morphometry as introduced for 3D trabecular bone analysis ${ }^{29}$. Moreover, the canal network was spatially decomposed into single elements and analyzed subsequently by using element-based morphometry as recently introduced ${ }^{31}$. For this reason, we further defined corresponding element-based indices, which are given as mean values over the total number of elements (marked with brackets $<>$ ). Amongst those averaged indices were mean canal volume $(<\mathrm{Ca} . \mathrm{V}\rangle)$, mean canal diameter $(<\mathrm{Ca} . \mathrm{Dm}>)$, mean canal length $(<\mathrm{Ca} . \mathrm{Le}>)$, and mean canal orientation $(<\mathrm{Ca} . \theta>)$ in relation to the femoral long axis. All cannular indices were computed in 3D space without any model assumptions. On the cellular level, the negative imprint of the cortical bone formed - additionally to the canal network - a cellular system or more precisely, the osteocyte lacunar system. A number of osteocyte lacunar indices were introduced in analogy to standard morphometry, including number of lacunae (N.Lc), lacuna number density (N.Lc/Ct.TV), lacuna volume (Lc.V), and lacuna volume density (Lc.V/Ct.TV). Moreover, we introduced the element-based mean lacuna volume as the ratio $<\mathrm{Lc} . \mathrm{V}\rangle=\mathrm{Lc} . \mathrm{V} / \mathrm{N}$.Lc. An illustration of murine cortical bone on the organ, tissue, and cellular level as well as how the cannular parameters are defined is given in Figure 3.

\section{Application}

To partition bone quality into its regulatory pathways, we chose a mouse model called little (C57BL/6J-Ghrhrit/J) wherein regulation of bone morphometry is independent of growth hormone (GH) and insulin-like growth factor-I (IGFI) ${ }^{32}$. More precisely, the little mouse strain carries a mutation in the growth hormone-releasing hormone receptor (Ghrhr), resulting in undetectable circulating GH, and serum IGF-I that is fixed at low, but detectable levels. For this study, we used homozygous mutant mice from the inbred strain C57BL/6J-Ghrhr $r^{l i t} / \mathrm{J}$ (B6-lit/lit) and the congenic strain C3.B6-Ghrhr lit/J (C3.B6-lit/lit) that was created by backcrossing the little mutation from the original B6 background to $\mathrm{C} 3 \mathrm{H}$. The result of introgressing the little mutation onto the $\mathrm{C} 3 \mathrm{H}$ background is that $\mathrm{C} 3$.B6-lit/lit mice have higher BMD than B6-lit/lit ${ }^{32}$. On necropsy, femora were dissected and subsequently stored in ethanol. All mice were raised at The Jackson Laboratory, and all animal procedures were approved by the Jackson Laboratory's Animal Care and Use Committee. 


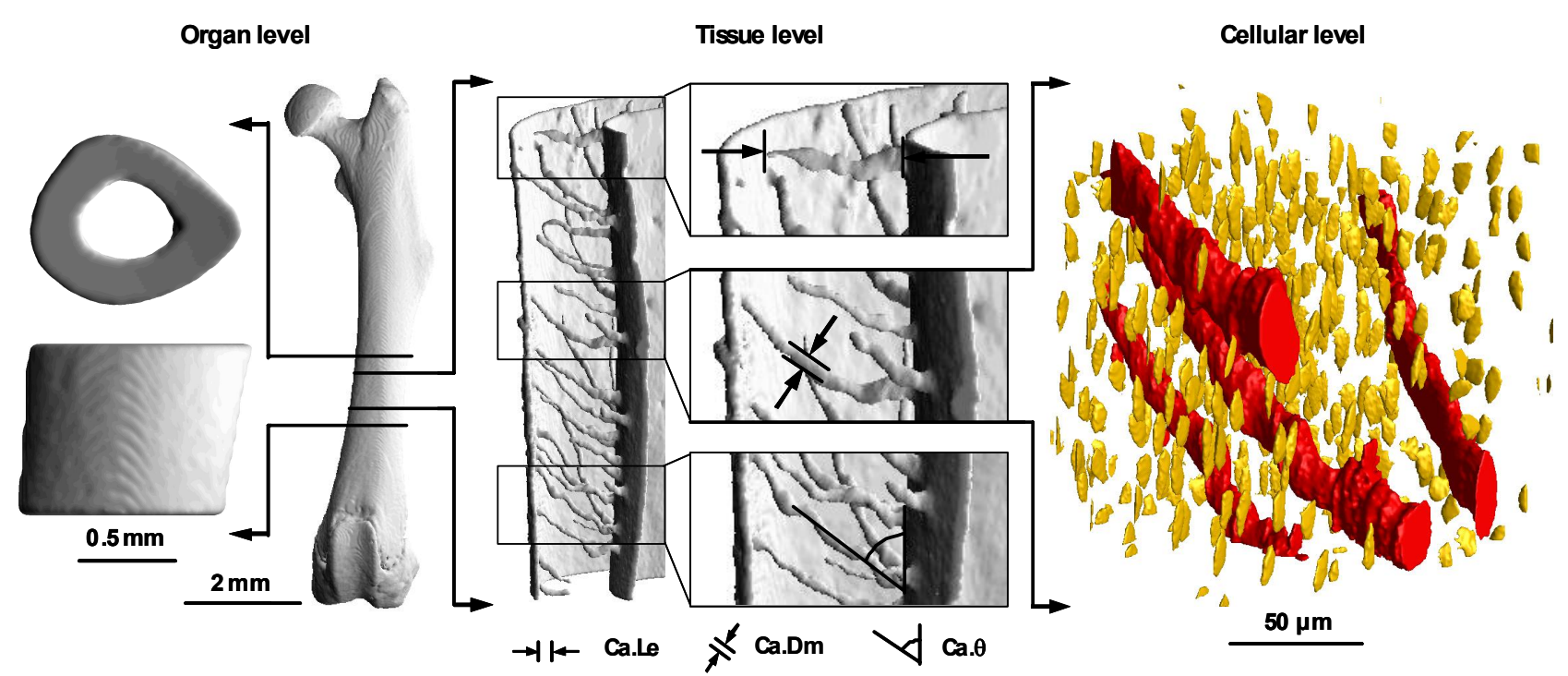

Figure 3. Hierarchical organization of murine cortical bone. Organ level: Murine femur with mid-diaphyseal compartment (indicated by the inner horizontal bars) that was measured at the Swiss Light Source (SLS). Tissue level: Illustration of the cannular parameters, which were introduced in the study for morphometry of the canal network. From top to bottom, the windows explain mean canal length $(<\mathrm{Ca} . \mathrm{Le}>)$, mean canal diameter $(<\mathrm{Ca} . \mathrm{Dm}>)$, and mean canal orientation $(<\mathrm{Ca} . \theta>)$. Data assessed by global SR CT at 3.5- $\mu \mathrm{m}$ nominal resolution. Cellular level: Canal network (tubes in red) and osteocyte lacunae (prolate ellipsoids in yellow) within cortical bone. Data assessed by local SR CT at 700-nm nominal resolution.

To study the organ and tissue level of the cortical bone, we scanned the femoral mid-diaphysis of 12 B6-lit/lit (6 female and 6 male) and 12 C3.B6-lit/lit (6 female and 6 male) 4-month-old mice using a conventional (i.e., global) SR CT setup at a nominal resolution of $3.5 \mu \mathrm{m}$. Because of the limited access to SR facilities, only two B6-lit/lit (one female and one male) and two C3.B6-lit/lit (one female and one male) femora were scanned locally, each at the anterior, posterior, lateral, and medial site of the mid-diaphysis. The FOV within the cortical bone assessed by the local SR CT measurements was a subset of the FOV of the global SR CT setup, so that the same cortical regions were imaged and corresponding ultrastructural morphometric parameters could be assessed.

For statistical analysis, the GNU statistical package R (Version 2.4.1, http://www.r-project.org) was used. The SR CT measurements on the organ and tissue hierarchy level comprised B6-lit/lit (6 female and 6 male) and C3.B6-lit/lit (6 female and 6 male) mice and consequently, our experimental setup represented a 2 × 2 factorial design, implying the two-level independent variables mouse strain (B6-lit/lit, C3.B6-lit/lit) and sex (female, male). A two-way ANOVA was performed to test for significances $(p<0.05)$. The primary osteocyte lacunar indices (N.Lc, Lc.V, and corresponding Ct.TV) were summed up over all sites (anterior, posterior, lateral, and medial) to calculate the lacunar ratios N.Lc/Ct.TV, Lc.V/Ct.TV, and $<$ Lc.V $>=$ Lc.V/N.Lc. No further statistics were performed for the cellular level because only one animal per mouse strain and sex was measured on this hierarchy level.

The morphometrical results for the cortical bone compartment (organ level) are summarized in Table 1, for the canal network (tissue level) in Table 2, and for the osteocyte lacunae (cellular level) in Table 3. 
Table 1: Cortical indices of B6-lit/lit and C3.B6-lit/lit mice on the organ level.

\begin{tabular}{|c|c|c|c|c|c|c|c|c|c|}
\hline \multirow[b]{2}{*}{ Parameter } & \multicolumn{3}{|c|}{ B6-lit/lit } & \multicolumn{3}{|c|}{ C3.B6-lit/lit } & \multicolumn{3}{|c|}{$A N O V A$} \\
\hline & Female & Male & $\operatorname{Diff}(\%)$ & Female & Male & $\operatorname{Diff}(\%)$ & Strain & Sex & Inter \\
\hline $\mathrm{TV}\left(\mathrm{mm}^{3}\right)$ & $1.47 \pm 0.07$ & $1.56 \pm 0.10$ & 6 & $1.15 \pm 0.07$ & $1.18 \pm 0.11$ & 3 & $<0.001$ & 0.098 & 0.409 \\
\hline Ct.BV $\left(\mathrm{mm}^{3}\right)$ & $0.60 \pm 0.04$ & $0.61 \pm 0.03$ & 3 & $0.76 \pm 0.06$ & $0.75 \pm 0.08$ & -2 & $<0.001$ & 0.982 & 0.494 \\
\hline Ct.BV/TV (\%) & $41 \pm 1$ & $39 \pm 2$ & -3 & $66 \pm 2$ & $63 \pm 2$ & -5 & $<0.001$ & $<0.01$ & 0.153 \\
\hline Ct.Th $(\mu \mathrm{m})$ & $134 \pm 7$ & $133 \pm 4$ & -1 & $222 \pm 12$ & $210 \pm 15$ & -6 & $<0.001$ & 0.124 & 0.197 \\
\hline $\mathrm{J}\left(\mathrm{mm}^{4}\right)$ & $0.13 \pm 0.02$ & $0.14 \pm 0.02$ & 10 & $0.10 \pm 0.01$ & $0.11 \pm 0.02$ & 3 & $<0.001$ & 0.286 & 0.491 \\
\hline
\end{tabular}

Values are mean $\pm \mathrm{SD}$.

Inter within ANOVA denotes interaction between the factors mouse strain and sex.

Total tissue volume (TV) including the medullary cavity and the cortical bone tissue, cortical bone volume (Ct.BV), cortical bone volume density (Ct.BV/TV), cortical thickness (Ct.Th), and polar area moment (J).

Table 2: Cannular indices of B6-lit/lit and C3.B6-lit/lit mice on the tissue level.

\begin{tabular}{|c|c|c|c|c|c|c|c|c|c|}
\hline \multirow[b]{2}{*}{ Parameter } & \multicolumn{3}{|c|}{ B6-lit/lit } & \multicolumn{3}{|c|}{ C3.B6-lit/lit } & \multicolumn{3}{|c|}{$A N O V A$} \\
\hline & Female & Male & Mean & Female & Male & Mean & Strain & Sex & Inter \\
\hline N.Ca/Ct.TV $\left(\mathrm{mm}^{-3}\right)$ & $197 \pm 45$ & $190 \pm 48$ & $193 \pm 44$ & $184 \pm 21$ & $142 \pm 27$ & $163 \pm 32$ & 0.060 & 0.126 & 0.256 \\
\hline Ca.V/Ct.TV (\%o) & $1.7 \pm 0.6$ & $1.8 \pm 0.8$ & $1.8 \pm 0.6$ & $5.7 \pm 0.8$ & $3.8 \pm 1.5$ & $4.8 \pm 1.5$ & $<0.001$ & $<0.05$ & $<0.05$ \\
\hline $\mathrm{Ca} . \mathrm{Sp}(\mu \mathrm{m})$ & $130 \pm 5$ & $129 \pm 4$ & $129 \pm 5$ & $179 \pm 7$ & $184 \pm 7$ & $181 \pm 7$ & $<0.001$ & 0.498 & 0.217 \\
\hline$<\mathrm{Ca} \cdot \mathrm{V}>\left(10^{3} \cdot \mu \mathrm{m}^{3}\right)$ & $10 \pm 1$ & $10 \pm 3$ & $10 \pm 2$ & $28 \pm 4$ & $20 \pm 1$ & $24 \pm 5$ & $<0.001$ & $<0.01$ & $<0.001$ \\
\hline$<$ Ca.Dm $>(\mu \mathrm{m})$ & $7.9 \pm 0.7$ & $7.8 \pm 0.9$ & $7.8 \pm 0.8$ & $9.3 \pm 0.6$ & $8.9 \pm 0.3$ & $9.1 \pm 0.5$ & $<0.001$ & 0.315 & 0.624 \\
\hline$<\mathrm{Ca} . \mathrm{Le}>(\mu \mathrm{m})$ & $71 \pm 8$ & $78 \pm 9$ & $74 \pm 9$ & $173 \pm 19$ & $128 \pm 12$ & $150 \pm 28$ & $<0.001$ & $<0.01$ & $<0.001$ \\
\hline$<\mathrm{Ca} . \theta>(\mathrm{deg})$ & $62 \pm 6$ & $55 \pm 8$ & $59 \pm 7$ & $36 \pm 4$ & $40 \pm 3$ & $38 \pm 4$ & $<0.001$ & 0.602 & $<0.05$ \\
\hline
\end{tabular}

Values are mean $\pm \mathrm{SD}$.

Inter within ANOVA denotes interaction between the factors mouse strain and sex.

Canal number density (N.Ca/Ct.TV), canal volume density (Ca.V/Ct.TV), canal spacing (Ca.Sp), mean canal volume

$(<\mathrm{Ca} . \mathrm{V}>)$, mean canal diameter $(<\mathrm{Ca} . \mathrm{Dm}>)$, mean canal length $(<\mathrm{Ca} . \mathrm{Le}>)$, and mean canal orientation $(<\mathrm{Ca} . \theta>)$.

Table 3: Osteocyte lacunar indices of B6-lit/lit and C3.B6-lit/lit mice on the cellular level.

\begin{tabular}{lcccccccc}
\hline & \multicolumn{3}{c}{ B6-lit/lit } & & \multicolumn{3}{c}{ C3.B6-lit/lit } \\
\cline { 2 - 4 } \cline { 7 - 8 } \multicolumn{1}{c}{ Parameter } & Female & Male & Mean & & Female & Male & Mean \\
\hline N.Lc/Ct.TV $\left(\mathrm{mm}^{-3}\right)$ & 68,257 & 63,473 & 65,865 & & 48,611 & 51,146 & 49,879 \\
Lc.V/Ct.TV $(\%)$ & 1.4 & 1.2 & 1.3 & & 1.3 & 1.4 & 1.3 \\
$<$ Lc.V $>\left(\mu \mathrm{m}^{3}\right)$ & 209 & 191 & 200 & & 265 & 274 & 269
\end{tabular}

Primary indices number of lacunae (N.Lc), lacuna volume (Lc.V), and corresponding cortical total volume Ct.TV, averaged over all sites (anterior, posterior, lateral, and medial). Ratios lacuna number density (N.Lc/Ct.TV), lacuna volume density (Lc.V/Ct.TV), and mean lacuna volume $(<$ Lc.V $>=$ Lc.V/N.Lc).

\section{Conclusions}

This is the first study investigating quantitatively the ultrastructural cortical bone morphometry of mice in 3D and in a fully non-destructive fashion. Murine intracortical porosity has been disentangled into the canal network and the osteocyte lacunar system, as two ultrastructural phenotypes of (murine) cortical bone. Global and local SR-based CT methods have been designed for quantification and morphometric analysis of these phenotypes. 


\section{STRUCTURE FUNCTION RELATIONSHIPS IN MURINE BONE}

\subsection{The influence of the cortical canal network on murine bone mechanics}

\section{Introduction}

Cortical geometry and particularly intracortical porosity have been demonstrated earlier to be linked to stiffness and strength of cortical bone specimens from human donors ${ }^{33-35}$ and from different mammals ${ }^{33,36,37}$. Furthermore, intracortical porosity has been associated with fracture risk of patients with femoral neck fractures ${ }^{38}$. Changes in human femoral intracortical porosity have been accounted for $76 \%$ of the reduction in bone strength ${ }^{39}$ and several other studies revealed the negative influence of increased intracortical porosity on fracture resistance ${ }^{40-42}$. Finally, we (section 2.2 ) and others $^{8-10}$ have shown that the canal network is a major contributor to local tissue porosity, and therefore, can directly be linked to measures of bone tissue quality and thus, to the mechanical properties of bone. On this account, the aim of this study was to provide evidence for a significant influence of the canal network on murine bone mechanics.

\section{Methods}

To study the canal network morphometry within murine cortical femoral bone, we used the two mouse strains C57BL/6J-Ghrhr $r^{l i t} / \mathrm{J}(\mathrm{B} 6-l i t /+)$ and C57BL/6J-Ghrhr $r^{l i t} / \mathrm{J}(\mathrm{C} 3 . \mathrm{B} 6-l i t /+)$, which are heterozygous for the little mutation ${ }^{32}$, in contrast to homozygous little mice known from the previous study presented in section 2.2 (Application). Since heterozygous little mice are expected to show GH-dependent mouse strain and sex differences in bone morphometry and bone mechanics, this should allow for the systematic study of structure function relationships within bone tissue and in particular, for quantifying the influence of the cortical canal network on murine bone mechanics. In total, 12 mice each (6 female and 6 male) of the two little mouse strains B6-lit/+ and C3.B6-lit/+ were used in this study. On necropsy at the age of 4 months, femora were dissected and subsequently stored in ethanol. All mice were raised at The Jackson Laboratory, and all animal procedures were approved by the Jackson Laboratory's Animal Care and Use Committee.

The femoral mid-diaphyses of all mice were scanned using a SR-based CT setup providing a nominal resolution of 3.5 $\mu \mathrm{m}$, as described previously in section 2.2 (Methods). For morphometry on the macroscopic level, tissue volume (TV) enclosing both trabecular bone within the medullary cavity and the cortical bone tissue, total bone volume (T.BV), total bone volume density (T.BV/TV) or apparent volume density (AVD) ${ }^{43}$, cortical thickness (Ct.Th), and polar area moment (J) were assessed. The cannular morphometric indices assessed on the tissue level are described in section 2.2 (Methods). For biomechanical testing, the contralateral femora of those scanned by SR CT were excised. Before testing, the bones were rehydrated for 24 hours in phosphate buffered saline (PBS). After a preload of $1 \mathrm{~N}$ the mouse femora were loaded to failure in anterior-posterior direction by three-point bending, using a custom-made loading device with a support distance of $5 \mathrm{~mm}$, which was integrated in a materials testing machine (1456; Zwick, Ulm, Germany). The femora were positioned so that the load was applied at $56 \%$ of the whole femur length (calculated from the greater trochanter), which corresponds to the femoral height where the SR CT measurements were performed. During three-point bending, the femora were lying freely and load-displacement curves were recorded at a crosshead speed of $0.5 \mathrm{~mm} / \mathrm{s}^{44}$. Ultimate force $\left(F_{u}\right)$, work to failure $(U)$, and stiffness $(S)$ were calculated from the load-displacement curves as described elsewhere ${ }^{45}$. $F_{u}$ indicates the strength of the bone, $S$ resistance to elastic deformation, and $U$ the required energy to fracture the bone.

For statistical analysis, the GNU statistical package R (Version 2.4.1, http://www.r-project.org) was used. Multiple linear regression models were built to explain the variation in the mechanical parameters $F_{u}, U$, and $S$ in terms of the morphometric indices. Models composed exclusively of macroscopic parameters were compared with mixed models, incorporating macroscopic and cannular indices as explanatory variables. Model selection was performed step-wise by backward-elimination, where the exclusion criterion for a specific morphometric parameter was based on Akaike's An Information Criterion $(A I C)^{46}$, its standardized regression coefficient $(\beta)$, significance, and degree of collinearity. $A I C$ is a measure of goodness of fit (the smaller the better) that penalizes models with too many parameters, while $\beta$ measures the influence of one morphometric index on the mechanical parameters. Further, the significance of one parameter was specified as $p$-value of a $t$-test $\left(p_{t}\right)$ for it's exclusion from the model and collinearity between the different morphometric indices was quantified by the Variance Inflation Factor $(V I F)^{47}$. Further, the global significance of one model was determined by the $p$-value of its $F$-statistic $\left(p_{F}\right)$. Explanatory variables were removed from a model in order of increasing $\beta$ and decreasing absolute differential $A I C$-values, where variables with $p_{t}<0.05$ were retained and insignificant models $\left(p_{F}>0.05\right)$ as well as final models including insignificant $\left(p_{t}>0.05\right)$ and highly collinear (VIF $\left.>5.0\right)$ variables were excluded. Resulting models were ranked according to their adjusted coefficient of determination $\left(R_{\text {adj }}^{2}\right)$ of the multiple linear regression and regarding their $A I C$-value, where models with highest $R_{a d j}^{2}$ and lowest $A I C$ were rated best. 


\section{Results}

The mechanical parameters $F_{u}, U$, and $S$ assessed from three-point bending are given in Table 4 .

Table 4: Mechanical parameters for B6-lit/+ and C3.B6-lit/+ mice.

\begin{tabular}{|c|c|c|c|c|c|c|c|c|}
\hline \multirow[b]{2}{*}{ Parameter } & \multicolumn{4}{|c|}{ B6-lit/+ } & \multicolumn{4}{|c|}{ C3.B6-lit/+ } \\
\hline & Female & Male & Mean & $\operatorname{Diff}(\%)$ & Female & Male & Mean & $\operatorname{Diff}(\%)$ \\
\hline$F_{u}(\mathrm{~N})$ & $18 \pm 2$ & $16 \pm 2$ & $17 \pm 2$ & -10 & $29 \pm 2$ & $35 \pm 3$ & $33 \pm 4$ & $21^{\mathrm{a}}$ \\
\hline$U(\mathrm{~mJ})$ & $3.3 \pm 1.2$ & $3.3 \pm 1.2$ & $3.3 \pm 1.1$ & 3 & $5.3 \pm 0.9$ & $7.2 \pm 1.7$ & $6.3 \pm 1.7$ & $37^{\mathrm{b}}$ \\
\hline$S(\mathrm{~N} / \mathrm{mm})$ & $179 \pm 33$ & $151 \pm 16$ & $165 \pm 29$ & -16 & $261 \pm 65$ & $313 \pm 59$ & $289 \pm 65$ & 20 \\
\hline
\end{tabular}

Values are mean \pm SD.

Unpaired $t$-test with Bonferroni correction: ${ }^{\mathrm{a}} p(\operatorname{sex})<0.01,{ }^{\mathrm{b}} p(\mathrm{sex})<0.1$.

Ultimate force $\left(F_{u}\right)$, work to failure $(U)$, and stiffness $(S)$.

Analysis of linear regressions between the morphometric and mechanical parameters revealed that the variation in $F_{u}$ and $S$ for B6-lit/+ mice could only be explained partially by one macroscopic parameter $\left(0.35 \leq R^{2} \leq 0.60\right)$ and that variation could not be explained at all for $U$. Similar to B6-lit/+, $F_{u}$ and $U$ of C3.B6-lit/+ were only explained to some extent by one macroscopic parameter $\left(0.38 \leq R^{2} \leq 0.91\right)$, and it was now stiffness $(S)$ that remained linearly unrelated to any single macroscopic parameter. For one typical case, the linear regression is depicted in Figure 4A.
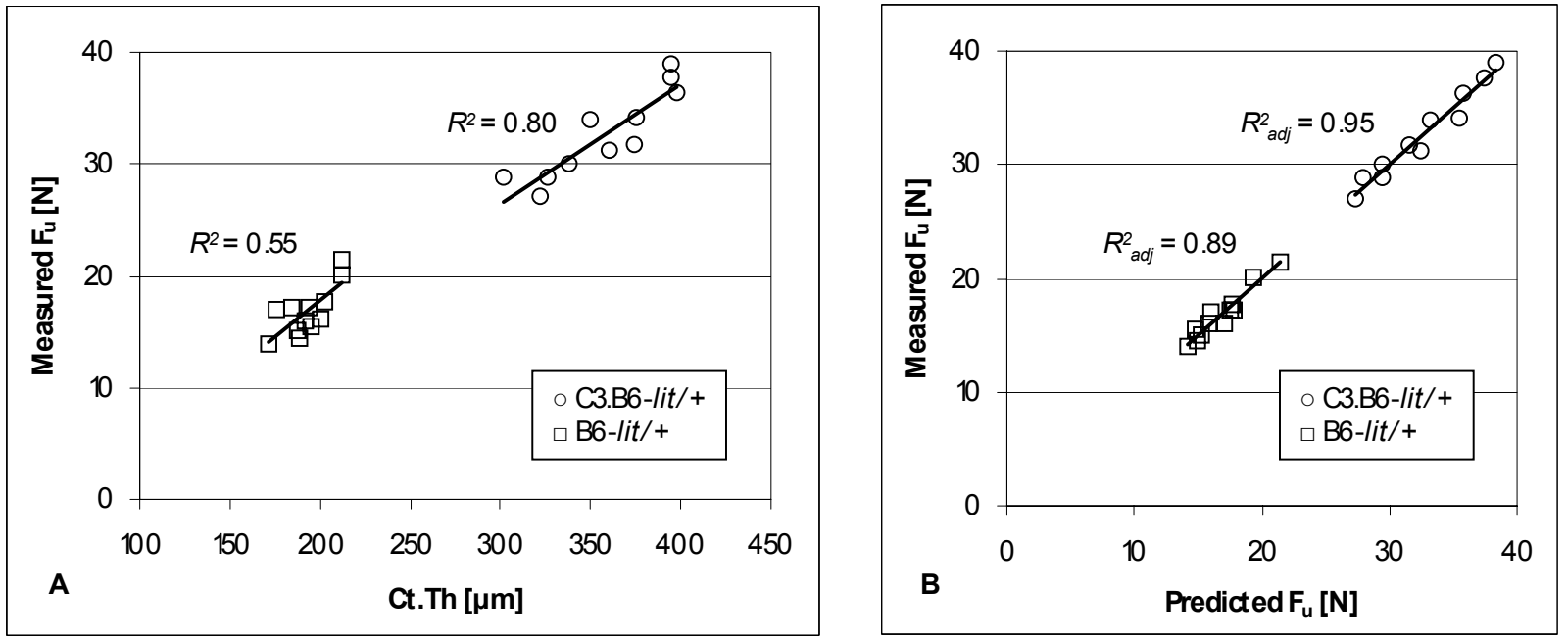

Figure 4. Bone strength prediction. $A$ : Macroscopic linear regression between cortical thickness (Ct.Th) and ultimate force $\left(F_{u}\right)$ of B6-lit/+ and C3.B6-lit/+ mice. B: Best mixed multiple linear models including macroscopic and cannular parameters for $F_{u}$ of B6-lit/+ and C3.B6-lit/+ mice. Compared with macroscopic linear regression (A), the explanatory power is enhanced, providing evidence for a significant influence of the canal network on murine bone mechanics.

To abstract the multiple linear regression results, we note first that for B6-lit/+ mice, no significant multiple linear models containing solely macroscopic parameters could be derived. Second and in contrast to the first point, mixed models could always be established for both mouse strains and in case where significant macroscopic models existed, they also had increased $R_{a d j}^{2}$ and lowered $A I C$-values, indicating that by using mixed models the explanatory power was improved and at the same time, no overfitting occurred. In numbers, $R_{a d j}^{2}$-values for B6-lit/+ mice were increased by $75 \%$ for $F_{u}$ and by $23 \%$ for $S$ comparing the respective best macroscopic and mixed model, whereas for $U$, the increase was virtually infinity because no significant corresponding macroscopic model existed. For C3.B6-lit/+, this increase in explanatory power was reduced to $6 \%$ for $F_{u}$ and to $117 \%$ for $U$, while now, the increase for $S$ was raised virtually to infinity. Comparing Figures $4 \mathrm{~A}$ and $4 \mathrm{~B}$ demonstrates the achieved improvements in explanatory power when rating the best models including macroscopic parameters only (Figure 4A) and models including cannular parameters in addition 
(Figure 4B): Predicted $F_{u}(\mathrm{~B} 6-\mathrm{lit} /+)[\mathrm{N}]=-1.6+0.227 \cdot \mathrm{Ct} . \mathrm{Th}[\mu \mathrm{m}]-0.141 \cdot<\mathrm{Ca} \cdot \mathrm{Le}>[\mu \mathrm{m}]-0.407 \cdot<\mathrm{Ca} . \theta>[\mathrm{deg}]+$ $0.57 \cdot<\mathrm{Ca} . \mathrm{V}>\left[10^{3} \cdot \mu \mathrm{m}^{3}\right]$ and predicted $F_{u}(\mathrm{C} 3 . \mathrm{B} 6-\mathrm{lit} /+)[\mathrm{N}]=13.0+36 \cdot \mathrm{J}\left[\mathrm{mm}^{4}\right]+0.042 \cdot \mathrm{Ca} \cdot \mathrm{Sp}[\mu \mathrm{m}]$.

\section{Conclusions}

The prediction power of the macroscopic morphometric parameters was increased significantly for all measured mechanical bone properties when including cannular parameters. We consequently provided strong evidence for a significant influence of the canal network on murine bone mechanics.

\section{CONCLUSIONS AND OUTLOOK}

We hypothesized that a better understanding of phenotypes linked to bone ultrastructure will provide new insight in the assessment of bone quality and its contribution to bone strength and fracture risk. To address this hypothesis, two specific aims were proposed for vascular and cellular imaging of bone (section 2) and for the examination of structure function relationships in murine bone (section 3).

For vascular and cellular imaging of bone, a SR-based local CT method in the submicrometer domain was developed for the non-destructive assessment and quantitative analysis of hard tissues. As a novelty, the devised CT method overcame present experimental limitations with respect to object size, currently precluding conventional CT methods from hard tissue assessment in the submicrometer regime for reasonably sized specimens. More generally, our strategy is applicable to new developments towards nanoCT and therefore, provides the prerequisite for 3D investigations of intact hard tissues at unprecedented resolutions. As one application, murine intracortical porosity has been disentangled into the canal network and the osteocyte lacunar system, as two ultrastructural phenotypes of (murine) cortical bone. SR CT methods have been designed for quantification of these phenotypes, based on newly devised morphometric indices characterizing the canal network and the osteocyte lacunar system within murine bone. We finally provided strong evidence that prediction of bone strength can be improved by including ultrastructural bone tissue properties.

As the technique of SR CT reaches the submicrometer domain, an objective for the future encompasses the quantitative assessment of microdamage, its initiation and propagation, to characterize another important determinant of bone quality and aspect of bone strength. Whereas this future work considers bone as a static system, the development of the established ultrastructural bone phenotypes over time is not addressed so far. Therefore, as a next step, the development of these phenotypes may be investigated over time, which will give a quantitative model for bone development down to a cellular level. Since there is the common hypothesis that the development of bone is governed by mechanical stimuli ${ }^{48}$,

the assessment of ultrastructural bone properties may be combined with recent mouse models for mechanotransduction ${ }^{49}$ to study bone adaptation in response to mechanical loading in vivo. Although the insight gained into the ultrastructure and biomechanics of murine bone may provide the requisite framework for genetic mouse studies dedicated to a better understanding of bone mechanics, the present knowledge may be transferred at one point to clinical practice. To this end, bone ultrastructure may be investigated in human studies in terms of fracture risk and incidence of osteoporosis.

\section{ACKNOWLEDGMENTS}

We would like to thank Dr. L. R. Donahue for providing the mouse specimens and Drs. M. R. Allen and D. B. Burr for the canine bone specimen. This study was supported by the Swiss National Science Foundation (SNF) through the SNF Professorship in Bioengineering (FP 620-58097.99 and PP-104317/1), by ETH Intramural Funding (TH 00124/412631.5) and by the United States Department of the Army (DAMD 17-01-1-0808).

\section{REFERENCES}

[1] Hui S. L., Slemenda C. W. and Johnston C. C., Jr., "Age and bone mass as predictors of fracture in a prospective study", J. Clin. Invest. 81(6), 1804-1809 (1988).

[2] Black D. M., Cummings S. R., Genant H. K., Nevitt M. C., Palermo L. and Browner W., "Axial and appendicular bone density predict fractures in older women", J. Bone Miner. Res. 7(6), 633-638 (1992).

[3] Nielsen S. P., "The fallacy of BMD: A critical review of the diagnostic use of dual X-ray absorptiometry", Clin. Rheumatol. 19(3), 174-183 (2000). 
[4] Ciarelli M. J., Goldstein S. A., Kuhn J. L., Cody D. D. and Brown M. B., "Evaluation of orthogonal mechanical properties and density of human trabecular bone from the major metaphyseal regions with materials testing and computed tomography", J. Orthop. Res. 9(5), 674-682 (1991).

[5] Müller R., "Bone microarchitecture assessment: current and future trends", Osteoporos. Int. 14(Suppl 5), S89-S99 (2003).

[6] Turner C. H., Cowin S. C., Rho J. Y., Ashman R. B. and Rice J. C., "The fabric dependence of the orthotropic elastic constants of cancellous bone", J. Biomech. 23(6), 549-561 (1990).

[7] Müller R., Hannan M., Smith S. Y. and Bauss F., "Intermittent ibandronate preserves bone quality and bone strength in the lumbar spine after 16 months of treatment in the ovariectomized cynomolgus monkey", J. Bone Miner. Res. 19(11), 1787-1796 (2004).

[8] Jones A. C., Sheppard A. P., Sok R. M., Arns C. H., Limaye A., Averdunk H., Brandwood A., Sakellariou A., Senden T. J., Milthorpe B. K. and Knackstedt M. A., "Three-dimensional analysis of cortical bone structure using Xray micro-computed tomography", Physica A 339(1-2), 125-130 (2004).

[9] Bousson V., Peyrin F., Bergot C., Hausard M., Sautet A. and Laredo J. D., "Cortical bone in the human femoral neck: three-dimensional appearance and porosity using synchrotron radiation", J. Bone Miner. Res. 19(5), 794-801 (2004).

[10] Cooper D. M., Turinsky A. L., Sensen C. W. and Hallgrimsson B., "Quantitative 3D analysis of the canal network in cortical bone by micro-computed tomography", Anat. Rec. B New Anat. 274(1), 169-179 (2003).

[11] Müller R., Van Campenhout H., Van Damme B., Van Der Perre G., Dequeker J., Hildebrand T. and Rüegsegger P., "Morphometric analysis of human bone biopsies: a quantitative structural comparison of histological sections and micro-computed tomography", Bone 23(1), 59-66 (1998).

[12] Martin T. and Koch A., "Recent developments in X-ray imaging with micrometer spatial resolution", J. Synchrotron Radiat. 13(2), 180-194 (2006).

[13] Snigireva I. and Snigirev A., "X-Ray microanalytical techniques based on synchrotron radiation", J. Environ. Monit. 8(1), 33-42 (2006).

[14] Prymak O., Tiemann H., Sotje I., Marxen J. C., Klocke A., Kahl-Nieke B., Beckmann F., Donath T. and Epple M., "Application of synchrotron-radiation-based computer microtomography (SRmicroCT) to selected biominerals: embryonic snails, statoliths of medusae, and human teeth", J. Biol. Inorg. Chem. 10(6), 688-695 (2005).

[15] Thurner P., Müller R., Raeber G., Sennhauser U. and Hubbell J., "3D Morphology of cell cultures: A quantitative approach using micrometer synchrotron light tomography", Microsc. Res. Tech. 66(6), 289-298 (2005).

[16] Donoghue P. C. J., Bengtson S., Dong X. P., Gostling N. J., Huldtgren T., Cunningham J. A., Yin C., Yue Z., Peng F. and Stampanoni M., "Synchrotron X-ray tomographic microscopy of fossil embryos", Nature 442(7103), 680-683 (2006).

[17] Stampanoni M., Borchert G., Wyss P., Abela R., Patterson B., Hunt S., Vermeulen D. and Rüegsegger P., "High resolution X-ray detector for synchrotron-based microtomography", Nucl. Instrum. Methods Phys. Res. A 491(1-2), 291-301 (2002).

[18] Patterson B. D., Abela R., Auderset H., Chen Q., Fauth F., Gozzo F., Ingold G., Kühne H., Lange M., Maden D., Meister D., Pattison P., Schmidt T., Schmitt B., Schulze-Briese C., Shi M., Stampanoni M. and Willmott P. R., "The materials science beamline at the Swiss Light Source: design and realization", Nucl. Instrum. Methods Phys. Res. A 540(1), 42-67 (2005).

[19] Kak A. C. and Slaney M., "Principles of computerized tomographic imaging", IEEE Press, New York, (1988).

[20] Faridani A., Buglione K. A., Huabsomboon P., Iancu O. D. and McGrath J. "Introduction to local tomography", In: Quinto E.T., Ehrenpreis L., Faridani A., Gonzalez F., and Grinberg E., editors, Radon transforms and tomography, American Mathematical Society, Providence, Rhode Island, 29-47 (2001).

[21] Chun I. K., Cho M. H., Lee S. C., Cho M. H. and Lee S. Y., "X-ray micro-tomography system for small-animal imaging with zoom-in imaging capability", Phys. Med. Biol. 49(17), 3889-3902 (2004).

[22] Shepp L. A. and Logan B. F., "The Fourier reconstruction of a head section", IEEE Trans. Nucl. Sci. 21(3), 21-43 (1974).

[23] Faridani A., Finch D. V., Ritman E. L. and Smith K. T., "Local tomography II", SIAM J. Appl. Math. 57(4), 10951127 (1997).

[24] Faridani A., Ritman E. L. and Smith K. T., "Examples of local tomography", SIAM J. Appl. Math. 52(4), 1193-1198 (1992).

[25] Currey J. D., "The many adaptations of bone", J. Biomech. 36(10), 1487-1495 (2003). 
[26] Schneider P., Stauber M., Voide R., Stampanoni M., Donahue L. R. and Müller R., "Ultrastructural properties in cortical bone vary greatly in two inbred strains of mice as assessed by synchrotron light based micro- and nano-CT", J. Bone Miner. Res. 22(10), 1557-1570 (2007).

[27] Kohler T., Beyeler M., Webster D. and Müller R., "Compartmental bone morphometry in the mouse femur: reproducibility and resolution dependence of microtomographic measurements", Calcif. Tissue Int. 77(5), 281-290 (2005).

[28] Leung C. K. and Lam F. K., "Performance analysis for a class of iterative image thresholding algorithms", Pattern Recognit. 29(9), 1523-1530 (1996).

[29] Hildebrand T., Laib A., Müller R., Dequeker J. and Rüegsegger P., "Direct three-dimensional morphometric analysis of human cancellous bone: microstructural data from spine, femur, iliac crest, and calcaneus", J. Bone Miner. Res. 14(7), 1167-1174 (1999).

[30] Parfitt A. M., Drezner M. K., Glorieux F. H., Kanis J. A., Malluche H., Meunier P. J., Ott S. M. and Recker R. R., "Bone histomorphometry: standardization of nomenclature, symbols, and units. Report of the ASBMR Histomorphometry Nomenclature Committee", J. Bone Miner. Res. 2(6), 595-610 (1987).

[31] Stauber M. and Müller R., "Volumetric spatial decomposition of trabecular bone into rods and plates - A new method for local bone morphometry", Bone 38(4), 475-484 (2006).

[32] Donahue L. R., Guido V. E., Rosen C. J., Horton L. G., Ackert-Bicknell C. L., Bouxsein M. L., Shultz K. L. and Beamer W. G., "GH/IGF-I independent genetic effects on BMD and skeletal morphology are both gender dependent and independent", J. Bone Miner. Res. 18(Suppl 2), S123 (2003).

[33] Saha S. and Hayes W. C., "Relations between tensile impact properties and microstructure of compact bone", Calcif. Tissue Res. 24(1), 65-72 (1977).

[34] Currey J. D., "Changes in the impact energy absorption of bone with age", J. Biomech. 12(6), 459-469 (1979).

[35] Dickenson R. P., Hutton W. C. and Stott J. R., "The mechanical properties of bone in osteoporosis", J. Bone Joint Surg. Br. 63-B(2), 233-238 (1981).

[36] Currey J. D., "The effect of porosity and mineral content on the Young's modulus of elasticity of compact bone", $J$. Biomech. 21(2), 131-139 (1988).

[37] Martin R. B. and Ishida J., "The relative effects of collagen fiber orientation, porosity, density, and mineralization on bone strength", J. Biomech. 22(5), 419-426 (1989).

[38] Barth R. W., Williams J. L. and Kaplan F. S., "Osteon morphometry in females with femoral neck fractures", Clin. Orthop. Relat. Res. 283, 178-186 (1992).

[39] McCalden R. W., McGeough J. A., Barker M. B. and Courtbrown C. M., "Age-related changes in the tensile properties of cortical bone. The relative importance of changes in porosity, mineralization, and microstructure", $J$. Bone Joint Surg. Am. 75(8), 1193-1205 (1993).

[40] Yeni Y. N., Brown C. U., Wang Z. and Norman T. L., "The influence of bone morphology on fracture toughness of the human femur and tibia", Bone 21(5), 453-459 (1997).

[41] Bell K. L., Loveridge N., Power J., Garrahan N., Meggitt B. F. and Reeve J., "Regional differences in cortical porosity in the fractured femoral neck", Bone 24(1), 57-64 (1999).

[42] Jordan G. R., Loveridge N., Bell K. L., Power J., Rushton N. and Reeve J., "Spatial clustering of remodeling osteons in the femoral neck cortex: a cause of weakness in hip fracture?" Bone 26(3), 305-313 (2000).

[43] Alexander J. M., Bab I., Fish S., Müller R., Uchiyama T., Gronowicz G., Nahounou M., Zhao Q., White D. W., Chorev M., Gazit D. and Rosenblatt M., "Human parathyroid hormone 1-34 reverses bone loss in ovariectomized mice", J. Bone Miner. Res. 16(9), 1665-1673 (2001).

[44] Turner C. H., Hsieh Y. F., Müller R., Bouxsein M. L., Baylink D. J., Rosen C. J., Grynpas M. D., Donahue L. R. and Beamer W. G., "Genetic regulation of cortical and trabecular bone strength and microstructure in inbred strains of mice", J. Bone Miner. Res. 15(6), 1126-1131 (2000).

[45] Turner C. H. and Burr D. B., "Basic biomechanical measurements of bone: a tutorial", Bone 14(4), 595-608 (1993).

[46] Akaike H., "New look at statistical-model identification", IEEE T. Automat. Contr. 19(6), 716-723 (1974).

[47] Marquard D. W., "Generalized inverses, ridge regression, biased linear estimation, and nonlinear estimation", Technometrics 12(3), 591-612 (1970).

[48] Robling A. G., Castillo A. B. and Turner C. H., "Biomechanical and molecular regulation of bone remodeling", Annu. Rev. Biomed. Eng. 8, 455-498 (2006).

[49] Warden S. J. and Turner C. H., "Mechanotransduction in the cortical bone is most efficient at loading frequencies of 5-10 Hz", Bone 34(2), 261-270 (2004). 